Exercices Exercices de rhétorique

de rhétorique $\quad 7 \mid 2016$

Les rhétoriques du peuple

\title{
En quarantaine : dissertation et auditoire [pseudo] universel
}

Françoise Collinet

\section{OpenEdition}

Journals

Édition électronique

URL : http://journals.openedition.org/rhetorique/474

DOI : $10.4000 /$ rhetorique.474

ISSN : 2270-6909

Éditeur

UGA Éditions/Université Grenoble Alpes

Édition imprimée

ISBN : 978-2-37747-084-6

Référence électronique

Françoise Collinet, «En quarantaine : dissertation et auditoire [pseudo] universel », Exercices de

rhétorique [En ligne], 7 | 2016, mis en ligne le 19 juin 2016, consulté le 12 septembre 2020. URL : http:// journals.openedition.org/rhetorique/474 ; DOI : https://doi.org/10.4000/rhetorique.474

Ce document a été généré automatiquement le 12 septembre 2020.

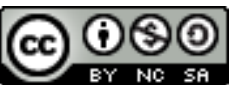

Les contenus de la revue Exercices de rhétorique sont mis à disposition selon les termes de la Licence Creative Commons Attribution - Pas d'Utilisation Commerciale - Partage dans les Mêmes Conditions 4.0 International. 


\title{
En quarantaine : dissertation et auditoire [pseudo] universel
}

\author{
Françoise Collinet
}

\section{Objectifs}

1 L'objectif du présent article est d'expérimenter, à travers un texte concret, des observations qui pourront par la suite être proposées dans un article plus théorique.

2 Le point de départ de notre propos peut ainsi être résumé : la dissertation s'est construite sur un refus de la rhétorique telle qu'on l'envisageait au XIX ${ }^{\mathrm{e}}$ siècle ; c'est-àdire une rhétorique des figures ou, comme le dit Genette ${ }^{1}$ une «rhétorique de l'elocutio ». Parmi les différentes formes qu'a prises, dans la seconde moitié du $\mathrm{XX}^{\mathrm{e}}$ siècle, la " résurrection de la rhétorique $^{2}$ ", la Nouvelle Rhétorique a pu apparaître comme une alternative pertinente à la dissertation qui pendant des décennies s'était présentée comme un refus de la rhétorique. Mais, surtout, à travers cette nouvelle légitimité de la rhétorique, c'est le statut antirhétorique de la dissertation qui perd de son évidence au point qu'elle finit par apparaître comme un avatar de la rhétorique. Ainsi, Genette requalifie-t-il la dissertation comme une rhétorique non assumée. De par son «statut idéologique ${ }^{3}$ ", cette rhétorique est une rhétorique " purement implicite ${ }^{4}$ » qui s'oppose à la rhétorique traditionnelle en ceci qu'au lieu d'être une rhétorique de l'elocutio, elle est une rhétorique de la dispositio ${ }^{5}$.

3 La Nouvelle Rhétorique, qui, pour compléter le classement de Genette, semble plutôt relever d'une rhétorique de l'inventio, brouille encore un peu plus le statut antirhétorique de la dissertation. Parce qu'elle se refuse à séparer le fond de la forme et enrôle les figures au service de l'argumentation ${ }^{6}$, la Nouvelle Rhétorique rend moins présente cette rhétorique traditionnelle contre laquelle s'était construite la dissertation mais qui, en même temps, fondait par contraste son identité. Ainsi, parce qu'il redéfinissait radicalement les frontières de l'Empire rhétorique, Perelman a indirectement contribué à transformer la dissertation en un objet étrange et paradoxal. 
4 À la fin des années '80, l'absence d'auditoire nettement identifiable, le discours antirhétorique de la dissertation et ses appels à la Raison, à la logique et à la clarté confirmaient le caractère obsolète et arbitraire du vieil exercice. Cette résistance de la dissertation au nouveau modèle rhétorique aurait cependant pu être posée en d'autres termes: la dissertation ne relève ni de la démonstration formelle (logique, mathématique) ni d'un discours intrinsèquement irrationnel, puisque le scripteur est supposé argumenter en raison. Elle devrait donc dépendre de la juridiction de cet Empire rhétorique dont Perelman a notablement étendu les territoires. Il faudrait dès lors admettre que la dissertation relève de ce vaste entre-deux auquel Perelman a étendu le territoire rhétorique et que le système descriptif devrait pouvoir traiter l'exception récalcitrante. Autrement dit, la dissertation, malgré son héritage antirhétorique et son insaisissable auditoire, devrait pouvoir s'analyser en termes perelmaniens.

5 Le paradoxe, en fait, n'est pas nouveau : les discours antirhétoriques ont, eux-mêmes, une dimension rhétorique ${ }^{7}$ et l'opposition entre ces discours est bien moins tranchée qu'on ne pourrait le penser de prime abord 8 .

6 La solution consisterait à utiliser deux concepts " régulateurs ${ }^{9}$ » qui, d'après nous, permettent de traiter avec souplesse un cas limite tel que la dissertation. La première notion est celle d'auditoire universel qui peut apparaitre comme une Incarnation de la Raison intemporelle ${ }^{10}$. Mais il ne faut pas oublier que, en même temps, pour Perelman, l'auditoire universel reste une fiction ${ }^{11}$. Derrière l'auditoire universel de droit auquel s'adresse le rédacteur d'une dissertation se cache un auditoire [pseudo]universel de fait : le discours dominant dans une société donnée à une époque donnée.

7 Le second concept régulateur est celui d'épidictique. Comme le rappelle Loïc Nicolas ${ }^{12}$, Perelman a accordé un rôle essentiel à l'épidictique qui, à première vue, s'intégrait mal à son système dans la mesure où il ne correspondait pas à la définition de sa théorie argumentative: on admet généralement que l'épidictique se caractérise par une absence de conflit, une volonté de gommer les tensions pour créer une communauté de sentiments ${ }^{13}$. Mais cette première difficulté, au contact d'un autre problème théorique, pouvait devenir un atout. En effet, les théories de l'argumentation présupposent un accord préalable: l'auditoire pourra éventuellement être convaincu mais pour cela, l'orateur devra prendre appui sur les convictions déjà acceptées par ces auditeurs afin de les mener, insensiblement, d'argument en argument, à des conclusions qu'ils n'auraient peut-être pas admises de but en blanc. L'épidictique permet de faire d'une pierre deux coups: il donne un statut rhétorique aux convictions préalables des individus tout en rendant à l'épidictique un enjeu sur le plan argumentatif. Cette transformation a cependant un prix : la rassurante classification d'Aristote fondée sur des situations sociales et des caractéristiques stylistiques nettement définies perd de sa clarté et la possibilité de genres hybrides ${ }^{14}$ autorise bien des scénarii. Mais peut-être cette nouvelle difficulté est-elle aussi une opportunité: la tradition relègue l'épidictique à des situations sociales bien circonscrites : l'éloge funèbre, les vœux du Chef de l'État, les discours d'inauguration, etc. On tente parfois d'élargir le cercle de ces situations convenues à des contextes plus familiers; on ajoute ainsi les cérémonies de fin d'année scolaire, les harangues des entraîneurs de football ${ }^{15}$. Peut-être des manifestations plus quotidiennes mais plus insidieusement efficaces devraient-elles relever de l'épidictique. Trois critères devraient permettre d'identifier une dimension épidictique. On songe d'abord à une forme de solennité ou de «presentness ${ }^{16}$ » qui agit 
sur les esprits (1). Au-delà de ses aspects techniques et plus ou moins rébarbatifs qui regardent les spécialistes, cette solennité est associée, par un ensemble d'individus donnés (2), à des valeurs dont cette communauté accepterait mal qu'elles soient remises en question (3) ; par exemple, dans une société comme la nôtre, l'état de droit, le droit de vote, la liberté de conscience, la présomption d'innocence mais aussi la réussite matérielle, la compétition, etc.

Un autre étonnement qu'on peut avoir face à la notion d'épidictique est que les commentateurs s'intéressent surtout à des situations de la vie adulte qui sont en marge du quotidien mais dont on pourrait par ailleurs considérer qu'elles réactivent souvent des discours entendus dans la jeunesse. Il vaudrait la peine de poser plus précisément la question de la dimension épidictique des discours adressés aux jeunes générations, notamment dans le cadre scolaire. C'est pourquoi nous proposons d'illustrer ce propos à travers une courte nouvelle ${ }^{17}$.

\section{Présentation de la Nouvelle et de l'extrait choisi au sein du récit}

9 En quarantaine est publié en 2001 par Jacqueline Harpman (1929-2012 $\left.{ }^{18}\right)$. La nouvelle compte, peut-être en hommage à son titre, une quarantaine de pages. Dans la mesure où la rédaction d'une dissertation constitue le nœud de l'intrigue, nous nous permettons de résumer la trame de l'histoire qui précède l'extrait étudié et lui donne tout son retentissement; nous aborderons également les complications entraînées par ce premier incident et certains commentaires qu'il suscite.

Par rapport à l'analyse d'un texte de dissertation proprement dit, cette nouvelle présente un intérêt non négligeable : avec le regard de la maturité, la narratrice revient sur les motivations qui pouvaient être celle d'une bonne élève de sa génération au moment où elle rédigeait une dissertation. Ces quelques décennies de décalage devraient également nous aider à repérer plus facilement des valeurs qui, sans nous être étrangères, ne correspondent pas parfaitement à notre époque ${ }^{19}$. Un autre attrait $\mathrm{du}$ dispositif est qu'il met en scène les réactions de différents acteurs du monde scolaire (élèves, enseignants, direction) et notamment celles de la narratrice qui s'interroge sur leurs éventuelles motivations cachées et reprend, en le commentant, le discours de l'institution scolaire. Ainsi, le passage à la fiction permet-il, d'une autre manière que l'étude des discours politiques, une inscription de la rhétorique dans un espace social donné 20 .

11 La narratrice est une dame d'un certain âge qui se penche sur son passé. Ses souvenirs la mènent à Casablanca, à la fin de la seconde guerre mondiale ; Harpman construit avec beaucoup de soin la chronologie de son récit. L'histoire commence, vraisemblablement, quelques semaines après l'offensive Von Rundstedt en décembre 1944 et janvier 1945 (E.Qu., 8) et se clôture à l'approche de l'été 1945, alors que la guerre est déjà terminée. La narratrice a alors les traits d'une jeune fille belge d'origine juive d'une quinzaine d'années. Quatre ans plus tôt (donc probablement à l'automne 1941 et, en tout cas, à l'époque de Vichy) la jeune fille avait été admise, malgré ses origines, au collège « Mers-Sultan » $(E . Q u ., 18,26)$ à la suite d'un malentendu qui convenait autant à sa mère qu'à la directrice de l'établissement (E.Qu., 25). Avec plus d'acuité que la jeune fille, la dame aux cheveux gris observe comment, avec l'arrivée des Américains à 
Casablanca (1942), le petit monde qui l'entoure est tranquillement passé de Pétain à de Gaulle « en s'accrochant à la Marseillaise pour assurer la continuité » (E.Qu., 46; voir aussi Harpman, 8, 14).

12 Cette découverte d'une forme d'hypocrisie et d'une distorsion entre les bons sentiments et une forme de bon sens constitue le fil rouge du récit. La jeune fille, d'une innocence qui, a posteriori, inquiète la vieille dame, ne soupçonne guère qu'il n'y a pas que les paroles dans les passions (E.Qu., 7). Là aussi, la duplicité est le maître mot : « [...] il fallait feindre l'ignorance. Le secret était dans le consensus : on faisait semblant de ne rien nous cacher et nous faisions mine d'y croire » (E.Qu., 19).

L'ennui de la guerre n'offrant pas beaucoup de dérivatifs, la narratrice constate avec une lucidité acérée que la vie de ces jeunes filles se ramenait à une seule aventure: "nous étions furieusement bonnes élèves » (E.Qu., 22). C'est qu'à l'école, elles avaient "les siècles pour lieux de promenades" (E.Qu., 21). Et la narratrice d'évoquer son enthousiasme pour les exercices scolaires, son goût pour les pièces de Racine (qui " dépeint la passion ») et celles de Corneille (qui « met tant de passion à soumettre son monde au devoir ", Harpman, 35). Au-delà de la transmission d'un savoir objectif, ces lieux communs aux lycéens français fonctionnent comme des connaissances partagées aussi en ce sens que le jeune esprit tend à s'identifier aux idées et aux événements retracés : « au cours d'Histoire, on m'avait fait prêter serment au Jeu de Paume, j'avais veillé la nuit du Quatre Août, voté la mort de Louis XVI et je m'étais battue sans-culotte à Valmy, l'État s'était séparé de l'Église et je n'avais signé qu'avec réticence le Concordat, pour des raisons politiques mais sans engager mon âme » (48). En d'autres termes, les personnages historiques, les illustres Ancêtres apparaissent comme autant de modèles à imiter et à recréer en soi ${ }^{21}$ (Perelman, 1979 : 134-137).

En filigrane apparaissent aussi des motivations dont les programmes scolaires ne parlent guère : la volonté de briller, d'obtenir une meilleure note que ses rivales, la jalousie bouillonnante lorsque ce n'est pas le cas (E.Qu., 10, 11, 15, 20, 28, 37).

15 Parmi les camarades de la narratrice, Henriette Serrano semble la rivale la plus dangereuse et la narratrice ne manque pas de nous signaler ses petites bassesses (ou ce qu'elle interprète comme tel). Elle s'agace en particulier des mines que prend Henriette lorsque, au moment où le professeur veut l'interroger, elle évoque opportunément son inquiétude pour son frère disparu au front. La situation est d'autant plus irritante que les professeurs choisissent alors la narratrice comme victime de substitution, ce qui a un effet regrettable sur les notes de la demoiselle en question (E.Qu., 10).

\section{Une dissertation qui vire de l'épidictique à la polémique}

16 La situation bascule à cause d'un commentaire de texte que nous assimilons à une dissertation dans la mesure où il mène à un débat d'idées. Les élèves ont eu à commenter cette citation de Charles Péguy: «Heureux ceux qui sont morts pour la terre charnelle - Heureux les épis mûrs et les blés moissonnés" (E.Qu., 11). Il est manifeste que les collégiennes ont lu la poésie mais le débat porte essentiellement sur ces vers; on peut supposer que, suivant une méthode assez classique, le texte a été commenté en classe et que les élèves sont ensuite invitées à donner leur avis sur ce fragment choisi. Ce sont surtout des expressions telles que «j'avais soutenu la thèse 
selon laquelle... » qui, malgré l'étiquette que la narratrice attribue à l'exercice scolaire (« explication de texte », Harpman, 11), nous semble autoriser le rapprochement avec la dissertation.

\subsection{Construction de l'exercice scolaire ou les masques de l'idéologie}

17 Avec le recul, la narratrice perçoit bien le caractère construit et progressif du discours enseignant qui mène de la citation d'Horace, ennoblie et protégée par les prestiges de la langue latine, à celle plus accessible, de Rouget de l'Isle et puis, enfin, à celle plus métaphorique, de Péguy.

18 Le thème qui paraîtrait un peu étrange à des lycéens d'aujourd'hui se comprend fort bien à la lumière de la situation historique : la communauté, en proie à la peur et consciente des sacrifices imposés aux familles, se resserre autour des valeurs associées à sa survie. A posteriori, la narratrice pose sur le texte de Péguy, l'étiquette "idéologie » (E.Qu., 11).

19 Pour autant qu'on puisse en juger, l'enthousiasme d'Henriette pour le poème de Péguy semble, par contraste, bien plus conforme à l'effet escompté même si pour les jeunes filles éloignées $\mathrm{du}$ front les réalités cachées derrière la poésie restent vraisemblablement dans le brouillard.

20 Les émotions de la narratrice (jalousie, soif de gloire littéraire) n'ont pas encore été suffisamment captées par l'institution scolaire pour véritablement l'empêcher de confondre «la fin» (l'héroïsme du citoyen) et la «conséquence » (la gloire scolaire annonciatrice de gloire littéraire); mais ces sentiments, la jeune fille ne serait sans doute pas prête à les avouer dans sa composition puisque la guerre et ses victimes ne peuvent décemment servir de moyen à l'ambition littéraire ${ }^{22}$. Ces élèves se retrouvent ainsi, mutatis mutandis, dans la situation de l'orateur antique dont il s'agit d'apprécier le talent littéraire mais qui ne doit pas faire de littérature ni ériger trop visiblement un monument à sa gloire personnelle.

\subsection{Nul n'est prophète en son pays}

21 Les évangiles (Matthieu 13, 57 ; Luc 4, 24) rapportent que Jésus de retour dans sa terre natale fut accueilli par les sarcasmes ou la méfiance: comment les habitants de Nazareth auraient-ils pu voir le Fils de Dieu sous les traits du fils du charpentier? Les Anciens exigeaient au contraire que l'orateur soit un homme de la Cité, un membre de la communauté, ce qui, justement, le distinguerait du prophète voire du poète ${ }^{23}$.

22 Au-delà du désaccord de surface, on retrouve la même interrogation: l'étranger dispose-t-il du même droit de parole concernant les affaires ou les valeurs de la Cité ? Peut-être voit-il ce que les gens du lieu ne peuvent percevoir ; mais peut-être aussi estil incapable de comprendre les véritables enjeux de ces valeurs, d'en ressentir le caractère indiscutable. C'est cette seconde option qui semble activée par l'enseignante : "Votre patriotisme est tiède, me dit-on, mais évidemment, vous n'êtes pas française " (E.Qu., 11). Ce qui, à la limite, aurait pu apparaître comme une concession devient, en raison notamment de la mauvaise note, une sanction. Tous les ingrédients semblent 
réunis pour que la jeune fille se retrouve dans la situation de l'atopos, celui qui ne partage pas les lieux de la cité24.

\subsection{Conflit sur les valeurs et malléabilité des notions}

Le problème apparaît aussi comme un conflit de valeurs ou, pour mieux dire, de hiérarchisation des valeurs. La jeune fille échoue à comprendre que le discours dissertatif est subordonné à d'autres enjeux que ceux qu'il explicite. La vieille dame est cependant capable d'analyser plus finement la situation.

Le fragment aboutit ainsi à ce que Perelman et Olbrechts-Tyteca ${ }^{25}$ appelleraient une " dissociation de notions » autour du terme " esprit ». Alors que jusque-là, cette notion était suffisamment floue pour susciter un éloge systématique, une incompatibilité est apparue. La narratrice oppose désormais «avoir de l'esprit» et «avoir mauvais esprit ». Et cette opposition aboutit à un renversement des valeurs. En termes perelmaniens, on dira que pour la communauté désormais cachée derrière le plus impersonnel des pronoms personnels (le On de la doxa), le conflit de valeurs se résout à travers un couple de notions " remanié26" par la narratrice : pour la communauté, on peut «avoir de l'esprit » à condition de ne pas se montrer subversif à l'égard d'une valeur incontestable, nécessaire à la survie du groupe; bref, à condition de ne pas s'égarer au-delà des limites du «sens commun» (avoir de l'esprit/ ne pas faire du mauvais esprit). Au contraire, pour la jeune fille, il est incompréhensible que la valeur " avoir de l'esprit " puisse être limitée par des considérations sociales ou une forme de tact (ne pas faire du mauvais esprit / avoir de l'esprit).

Sur le même modèle, la narratrice oppose les bons sentiments/le bon sens. En opposant ainsi bon sens et bons sentiments, elle commence à discriminer ce qui, dans le discours scolaire, relève du logos et ce qui relève d'une forme de pathos qu'elle ne parvient pas à partager. On est aussi en droit de supposer que la petite communauté scolaire, tendue par les angoisses de la guerre, n'accepterait pas de définir le bon sens par «ne mourir que fort vieille, riche s'il se peut pour faire plaisir à mes héritiers, et comblée de plaisirs » (E.Qu., 13).

\subsection{Les limites du débat et [pseudo]-auditoire universel}

Comme le constate la narratrice, le désaccord se lit également comme une querelle portant sur les frontières assignées au débat possible : «On m'avait appris que deux et deux font quatre sans me dire qu'il ne fallait s'en servir que là où c'était prescrit » (E.Qu., 12).

C'est ce qui explique que le débat d'idées et la prise de position du candidat, en principe typiques de la dissertation, n'excluent pas la présence d'une dimension épidictique. Selon Beale ${ }^{27}$, l'épidictique n'est pas forcément incompatible avec une discussion sur des dichotomies ; à condition cependant que ces dichotomies ne soient pas réellement polarisantes (au sens de Ruth Amossy ${ }^{28}$ ). Le problème que suscite la dissertation de la narratrice est que contrairement aux devoirs habituels, la dichotomie qu'elle croit pouvoir utiliser est interdite du fait de son caractère polarisant. Et la narratrice de souligner: "Je pouvais avoir toutes les opinions que je voulais sur Rastignac ou sur Frédéric Moreau, on notait ma copie selon la correction du style et de l'orthographe " (E.Qu., 49) mais lorsqu'il s'est agi des «Héros de la Patrie » (E.Qu., 49) le champ des 
débats possibles s'est soudain restreint et derrière la Raison universelle est apparue l'institution. En termes perelmaniens :

L'auditoire universel auquel on est censé s'adresser coïncide, en fait, avec un auditoire particulier que l'on connaît et qui transcende les quelques oppositions dont on a actuellement conscience ${ }^{29}$.

\section{Du conflit de valeurs au « dialogue de sourds »}

La discussion, qui aurait pu en rester là, se prolonge après les leçons mais, au lieu de conduire à l'accord pourtant souhaité, le fossé entre la narratrice et Henriette puis la communauté, va irrémédiablement se creuser pour aboutir à un désaccord toujours plus profond où l'argumentation «normale » cesse d'être possible ${ }^{30}$. Alors même qu'au niveau des valeurs, un accord préalable ${ }^{31}$ semblerait globalement possible, le conflit quant à la hiérarchie des valeurs communes bloque toute conciliation et la colère, cette émotion d'autant plus redoutable qu'elle est toujours perçue comme légitime par celui qui l'éprouve, s'exacerbe ${ }^{32}$. Un débat installé dans ce genre d'évidences non partagées est de nature à s'inscrire dans la durée : la narratrice, incrédule, doit ainsi constater que, 50 ans plus tard, non seulement sa rancune est toujours là (E.Qu., 24) mais qu'en plus, elle tiendrait bien encore "sept fois sept générations " (E.Qu., 24) et le récit se conclut sur cette phrase lapidaire : «Je déteste Charles Péguy » (E.Qu., 51).

\subsection{D'une dispute entre camarades aux sanctions institutionnelles}

Les arguments de la narratrice ne sont pas exactement de nature à apaiser le conflit : la jeune fille s'étonne que sa camarade puisse penser que mourir pour la Patrie soit un bonheur et, en même temps, s'inquiéter pour son frère disparu au front ; Perelman et Olbrechts-Tyteca ${ }^{33}$ parleraient d'incompatibilité. Par ailleurs, les soldats allemands et catholiques devraient, eux aussi, avoir leur place au Paradis (E.Qu., 15, 16, 27) ; ce qui correspond à la règle de justice ${ }^{34}$.

Le personnage d'Henriette permet d'effleurer un des mécanismes de la "délation » (E.Qu., 29) : le dénonciateur signale un contrevenant à une autorité supposée légitime. Comme y insiste la narratrice, la dispute prend alors un caractère public (E.Qu., 30, 32) et la machine administrative se met en marche : "tout l'appareil scolaire me toisait » (E.Qu., 38).

31 La jeune fille refuse, à son propre étonnement d'ailleurs (E.Qu., 36-43), de présenter des excuses, de «se ranger avec la collectivité, tomber d'accord avec le plus grand nombre, et donc respecter le sentiment d'allégeance à la citée ${ }^{35}$ ». Et la sanction devient inévitable: la quarantaine. Autrement dit, la collégienne devra assister aux cours et accomplir toutes les tâches scolaires mais personne ne lui adressera la parole durant quarante jours.

\subsection{En quarantaine et spirale du silence ${ }^{36}$}

Le titre prend alors tout son sens : outre le fait que le récit évoque les années quarante, la jeune fille est mise en quarantaine mais on peut aussi considérer cette traversée du désert comme un temps de préparation, peut-être celui de l'écriture ou celui de la maturité. 

l'empêcher de contaminer le troupeau par ses idées subversives. La procédure rappelle également la mesure d'ostracisme de la Cité grecque. Par ailleurs, la narratrice se retrouve emmurée, pour quarante jours, dans le silence à la façon d'une Antigone moderne pour avoir voulu respecter « les principes mêmes » de la Communauté (E.Qu., $33,38,39$ ). Plus proche sur ce point d'Anouilh que de Sophocle, la narratrice consent, en bout de course, à humaniser ces enseignantes qu'elle a obligées à être cruelles (E.Qu., $26,40,42,43)$. deux sens ; la jeune fille refuse de sortir du silence : «c'était mon tour : je les mis toutes en quarantaine. Elles n'y comprenaient rien » $(E . Q u ., 45)$. Les enseignantes ont été humanisées aussi en ce sens qu'elles ont perdu leur prestige pour devenir des fonctionnaires de l'État soumises aux conventions sociales "pour gagner leur vie» (E.Qu., 47). Le discours scolaire qui semblait au service de la Vérité est devenu la doxa d'une petite communauté recroquevillée sur ses certitudes. Face au groupe et à ce qu'elle considère comme une injustice, la jeune rebelle pourrait faire figure d'«héroïne» (E.Qu., 47) témoignant d'une forme d'aristocratie de l'esprit adressée désormais à un auditoire plus restreint ${ }^{37}$. Peut-être Pernot ${ }^{38}$ n'a-t-il pas tort d'établir un lien entre la condamnation de Socrate par un jury populaire et la haine de Platon pour la rhétorique ou la démocratie.

35 Mais peut-on être Socrate sans la ciguë ? Avec la fin de l'année scolaire, qui correspond aussi à la fin de la guerre, une nouvelle vie, loin de Casablanca, va commencer. Les années semblent d'ailleurs avoir eu raison de la posture héroïque qui avait pu être celle de la jeune fille : « en suis-je à me souvenir de mes quinze ans et de mon silence enragé comme des anciens combattants qui brandissent toujours leur courage d'il y a cinq décennies et filent doux au moindre obstacle?» (E.Qu., 46). Et la dame de tempérer : «J'avais découvert la solitude et l'orgueil, ce qui était de mon âge » (E.Qu., 46).

\section{Conclusion}

Au-delà de son caractère fictif (et/ou autobiographique) qui a priori semblerait offrir peu de prise à l'analyse rhétorique d'un genre « réel » tel que la dissertation, le recours à une nouvelle telle que En quarantaine présente certains avantages. Sa construction narrative relativement complexe permet une analyse rhétorique à trois niveaux différents :

Niveau 1: La nouvelle offre une analyse de l'exercice proprement dit dont, il est vrai, le lecteur n'a qu'un écho. Mais peut-être parce que ce type d'exercice est-il suffisamment partagé pour permettre au lecteur de se faire une idée du problème évoqué.

Niveau 2: L'étude d'une dissertation attestée ne permettrait pas d'observer l'insertion de l'exercice dans un contexte social-historique particulier: la manière dont se construit le discours enseignant; la description de motivations que les élèves ne peuvent expliciter dans leurs textes. Le personnage d'Henriette permet de décrire le système de récompenses associées à la production du comportement attendu. Symétriquement, le personnage de la narratrice permet d'illustrer le système de sanctions croissantes qui se met en place lorsqu'une infraction mineure se transforme 
progressivement en un dialogue de sourds entre un individu et l'institution dont il dépend.

Niveau 3 : Le regard de la narratrice âgée démonte, à froid, la situation sociale décrite au niveau 2 et s'interroge sur les motivations des différents intervenants, y compris le positionnement final de la jeune rebelle qui pourrait n'être qu'un autre piège du moi ou des autres.

Telle Candide chassé du paradis de Thunder-ten-Tronck, la narratrice considère que cette aventure l'a " jetée à grands coups de pieds au cul hors de l'enfance » (E.Qu., 49). À certains égards, l'apprentissage aura consisté à identifier derrière l'auditoire universel construit par l'institution scolaire du temps, un [pseudo] auditoire universel de fait et à se rendre compte que le débat autorisé par l'institution scolaire recelait aussi une dimension épidictique dont il était alors inconvenant de déranger l'unanimité :

Le fait que l'épidictique est destiné à promouvoir des valeurs sur lesquelles on s'accorde, explique que l'on éprouve l'impression d'un abus lorsque, à l'occasion d'un pareil discours, quelqu'un prend position dans une matière controversée, détourne son argumentation vers des valeurs contestées, introduit des dissonances dans une circonstance créée pour favoriser la communion ${ }^{39}[. .$.$] .$

\section{BIBLIOGRAPHIE}

Amossy Ruth, 2014, Apologie de la polémique, Paris, Puf.

Angenot Marc, 2008, Dialogues de sourds : Traité de rhétorique antilogique, Paris, Mille et une Nuits.

Beale Walter H., 1978, « Rhetorical Performative Discourse : A new theory of Epideictic ", Philosophy \& Rhetoric, 11 / 4, 221-246 (URL : http://www.jstor.org/stable/40237084; consulté le 16.01.16).

Cassin Barbara, 1999, « Bonnes et mauvaises rhétoriques : de Platon à Perelman », Meyer Michel \& Lempereur Alain (dir.), Figures et conflits rhétoriques, Bruxelles, éds. de l'Université.

Collinet Françoise, 2014, « La dissertation française : un genre argumentatif entre rhétorique et logique (évidences, présupposés et changements de paradigme) », Romanica Cracoviensia, Kraków, 95-111 (DOI : 10.4467/20843917RC.13.008.2710).

Compagnon Antoine, 1986, « Martyre et résurrection de Sainte Rhétorique », Cassin Barbara (éd.), Le plaisir de parler, Paris, Minuit, 157-172.

Danblon Emmanuelle, 2004, « La Nouvelle Rhétorique de Perelman et la question de l'auditoire universel », Meyer Michel (éd.), Perelman, le renouveau de la rhétorique, Paris, Puf, 21-37.

Danblon Emmanuelle, 2002, Rhétorique et rationalité. Essai sur l'émergence de la critique et de la persuasion, Bruxelles, éds. de l'Université.

Danblon Emmanuelle, 2001, «La rationalité du discours épidictique », Dominicy Marc \& Frédéric Madeleine (éds.), La mise en scène des valeurs. La rhétorique de l'éloge et du blâme, Lausanne, Delachaux \& Niestlé, 19-48. 
Fogelin Robert J., 1985, « The logic of deep disagreements ", Informal Logic, 7, 1-8. URL : http:// ojs.uwindsor.ca/ojs/leddy/index.php/informal_logic/article/viewFile/1040/635, consulté le 14 janvier 2016.

Genette Gérard, 1969 [1966], « Rhétorique et enseignement », Figures II, Paris, Seuil, 23-42. Harpman Jacqueline, 2001, En quarantaine, Paris, Mille et une nuits.

Herman Thierry, 2011, « Manifestations rhétoriques des frontières idéologiques : le cas des minarets helvétiques », Semen, 30,127-146. URL : http://semen.revues.org/9003, consulté le 14 janvier 2016.

Hesk Jon, 1999, « The Rhetoric of Anti-rhetoric in Athenian oratory », Performance Culture and Athenian Democracy, Cambridge, 201-230. URL : https://www.academia.edu/920558/

The_Rhetoric_of_Anti-rhetoric_in_Athenian_oratory_pp._201230_in_Performance_Culture_ and_Athenian_Democracy_edited_by_Simon_Goldhill_and_Robin_Osborne_Cambridge_University_Press_1999_; consulté le 16.01.2016.

Kreutz Philippe, 2001, "L'épidictique et les émotions », Dominicy Marc et Frédéric Madeleine (éds.), La mise en scène des valeurs. La rhétorique de l'éloge et du blâme, Lausanne, Delachaux et Niestlé, 107-134.

Meyer Michel, 2010 [2008], Principia Rhetorica. Une théorie de l'argumentation, Paris, Puf.

Nicolas Loïc, 2015, « L'épidictique, assise et pivot de l'édifice rhétorique », Rivista Italiana di Filosofia del Linguaggio, 33-47.

Perelman Chaïm, 2012, « Logique et rhétorique » [1950], Rhétoriques [articles publiés entre 1945 et 1969] (Bruxelles, éds. de l'Université).

Perelman Chaïm, 1979 [1951], « Role of the model in education », The New Rhetoric and the Humanities. Essays on Rhetoric and its applications, Dordrecht, Reidel, 134-137.

Perelman Chaïm et Olbrechts-Tyteca Lucie, 2008 [1958], Traité de l'argumentation. La nouvelle rhétorique, Bruxelles, éds. de l'Université.

Pernot Laurent, 2000, La rhétorique dans l'Antiquité, Paris, Librairie générale française.

Ziomek Jerzy, 1990, Retoryka opisowa, Wrocław, Zakład Narodowy im. Ossolińskich.

\section{NOTES}

1. G. Genette, « Rhétorique et enseignement », Figures II, Paris, Seuil, 1969 [1966], p. 25-27.

2. A. Compagnon, « Martyre et résurrection de Sainte Rhétorique » dans B. Cassin éd., Le plaisir de parler, Paris, Minuit, 1986, p. 157-172.

3. G. Genette, op. cit., p. 28. L'auteur souligne.

4. Ibid.

5. Ibid., p. 31.

6. C. Perelman et L. Olbrechts-Tyteca, 2008 [1958], Traité de l'argumentation. La nouvelle rhétorique, Bruxelles, éd. de l'Université, p. 192 et 232.

7. J. Hesk, "The Rhetoric of Anti-rhetoric in Athenian oratory ", Performance Culture and Athenian Democracy, Cambridge, 1999, p. 201-230. URL: https://www.academia.edu/920558/ The_Rhetoric_of_Anti-

rhetoric_in_Athenian_oratory_pp._201230_in_Performance_Culture_and_Athenian_Democracy_edited_by_Simon_Goldhill_and_Robi ; consulté le 16.01.2016. 
8. L Pernot, La rhétorique dans l'Antiquité, Paris, Librairie générale française, 2000, p. 74-79.

9. B. Cassin, «Bonnes et mauvaises rhétoriques: de Platon à Perelman», M. Meyer et A. Lempereur dir., Figures et conflits rhétoriques, Bruxelles, éds. de l'Université, 1999, p. 33 ; E. Danblon, «La Nouvelle Rhétorique de Perelman et la question de l'auditoire universel », M. Meyer éd., Perelman, le renouveau de la rhétorique, Paris, Puf, 2004, p. 25.

10. M. Meyer, Principia Rhetorica. Une théorie de l'argumentation, Paris, Puf, 2010 [2008], p. 61.

11. C. Perelman, « Logique et rhétorique » [1950], Rhétoriques [Articles publiés entre 1945 et 1969], Bruxelles, éds. de l'Université, 2012, p. 76 et 77 ; M. Meyer, op. cit., p. 162.

12. L. Nicolas, «L'épidictique, assise et pivot de l'édifice rhétorique ", Rivista Italiana di Filosofia del Linguaggio (RIFL), 2015, p. 33-47.

13. L. Pernot, La rhétorique de l'éloge dans le monde gréco-romain, Paris, Institut d'Études Augustiniennes, 1993, p. 720-721 tel que cité par E. Danblon, «La rationalité du discours épidictique ", M. Dominicy \& M. Frédéric éd., La mise en scène des valeurs. La rhétorique de l'éloge et du blâme, Lausanne, Delachaux \& Niestlé, 2001, p. 29.

14. W. H. Beale, «Rhetorical Performative Discourse : A new theory of Epideictic », Philosophy \& Rhetoric, 11/4, 1978, p. 235. URL : http://www.jstor.org/stable/40237084; consulté le 16.01.16.

15. E. Danblon, « La rationalité du discours épidictique », op. cit., p. 20.

16. W. H. Beale, op. cit., p. 224.

17. Nous nous sommes déjà servie de ce texte par le passé : F. Collinet, «La dissertation française: un genre argumentatif entre rhétorique et logique (évidences, présupposés et changements de paradigme)», Romanica Cracoviensia, Kraków, 2014, 95-111, DOI : 10.4467/20843917RC.13.008.2710. Il s'agit, sous un angle différent, d'une relecture plus complète du propos antérieur.

18. J. Harpman, En quarantaine, Paris, Mille et une nuits, 2001. Nous donnerons les références de pagination dans le fil de notre propos.

19. C. Perelman et L. Olbrechts-Tyteca, op. cit., p. 223.

20. T. Herman, «Manifestations rhétoriques des frontières idéologiques: le cas des minarets helvétiques », Semen, 30, 127-146, § 6. URL : http://semen.revues.org/9003, consulté le 14 janvier 2016.

21. C. Perelman, "Role of the model in education ", The New Rhetoric and the Humanities. Essays on Rhetoric and its applications, Dordrecht, Reidel, 1979 [1951], p. 134-137.

22. C. Perelman, « Logique et rhétorique », op. cit., p. 71.

23. J. Ziomek, Retoryka opisowa, Wrocław, Zakład Narodowy im. Ossolińskich, 1990, p. 10.

24. T. Herman, op. cit., § 5 ; E. Danblon, Rhétorique et rationalité. Essai sur l'émergence de la critique et de la persuasion, Bruxelles, éds. de l'Université, 2002, p. 31.

25. Op. cit., p. 550-555.

26. Ibid., p. 551.

27. Op. cit., p. 238.

28. R. Amossy, Apologie de la polémique, Paris, Puf, 2014, p. 55.

29. C. Perelman, « Logique et rhétorique », op. cit., p. 77.

30. R. J. Fogelin, «The logic of deep disagreements », Informal Logic, 7, 1985, p. 7, 8 ; URL : http:// ojs.uwindsor.ca/ojs/leddy/index.php/informal_logic/article/viewFile/1040/635, consulté le 14 janvier 2016. Voir aussi M. Angenot, Dialogues de sourds : Traité de rhétorique antilogique, Paris, Mille et une Nuits, 2008, p. 11.

31. C. Perelman et L. Olbrechts-Tyteca, op. cit., p. 87-88.

32. P. Kreutz, «L'épidictique et les émotions », M. Dominicy \& M. Frédéric dir., op. cit., p. 123.

33. Op. cit., p. 262.

34. C. Perelman \& L. Olbrechts-Tyteca, op. cit., p. 294.

35. E. Danblon, «La rationalité du discours épidictique », op. cit., p. 29. Voir aussi T. Herman, op. cit., § 5 . 
36. Cette expression est empruntée à Noelle-Neuman, Elisabeth, Öffentliche Meinung: die Entdeckung der Schweigespirale, Frankfurt am Main, Ullstein, 1989 ; citée par T. Herman, op. cit., $\S 29$.

37. C. Perelman, « Logique et rhétorique », op. cit., p. 77.

38. L. Pernot, op. cit., p. 68-69.

39. C. Perelman \& L. Olbrechts-Tyteca, op. cit., p. 70.

\section{AUTEUR}

\section{FRANÇOISE COLLINET}

Université Jagellonne, Cracovie 